\title{
The Double-peaked Radio Light Curve of Supernova PTF11qcj
}

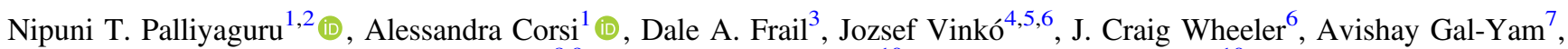 \\ S. Bradley Cenko ${ }^{8,9}$, Shrinivas R. Kulkarni ${ }^{10}$, and Mansi M. Kasliwal ${ }^{10}$ \\ ${ }^{1}$ Department of Physics and Astronomy, Texas Tech University, Box 41051, Lubbock, TX 79409, USA; nipunipalliyaguru9@gmail.com \\ 2 Arecibo Observatory, HC3 Box 53995, Arecibo, PR 00612, USA \\ ${ }^{3}$ National Radio Astronomy Observatory, 1003 Lopezville Road, Socorro, NM 87801, USA \\ ${ }^{4}$ Konkoly Observatory, Research Centre for Astronomy and Earth Sciences, Hungarian Academy of Sciences, Konkoly-Thege M. út 15-17, Budapest, 1121, Hungary \\ ${ }^{5}$ Department of Optics and Quantum Electronics, University of Szeged, Dómtér 9, Szeged, 6720, Hungary \\ ${ }^{6}$ Department of Astronomy, University of Texas at Austin, Austin, TX 78712, USA \\ ${ }^{7}$ Benoziyo Center for Astrophysics, Weizmann Institute of Science, 76100 Rehovot, Israel \\ ${ }^{8}$ Astrophysics Science Division, NASA Goddard Space Flight Center, Mail Code 661, Greenbelt, MD 20771, USA \\ ${ }^{10}$ Division of Physics, Math and Astronomy, California Institute of Technology, 1200 East California Boulevard, Pasadena, CA 91125, USA \\ Received 2018 June 10; revised 2018 November 22; accepted 2018 December 3; published 2019 February 25
}

\begin{abstract}
We present continued radio and X-ray follow-up observations of PTF11qcj, a highly energetic broad-lined Type Ic supernova (SN), with a radio peak luminosity comparable to that of the $\gamma$-ray burst (GRB) associated SN $1998 \mathrm{bw}$. The latest radio observations, carried out with the Karl G. Jansky Very Large Array, extend up to $\sim 5$ yr after the PTF11qcj optical discovery. The radio light curve shows a double-peak profile, possibly associated with density variations in the circumstellar medium (CSM), or with the presence of an off-axis GRB jet. Optical spectra of PTF11qcj taken during both peaks of the radio light curve do not show the broad $\mathrm{H} \alpha$ features typically expected from H-rich circumstellar interaction. Modeling of the second radio peak within the CSM-interaction scenario requires a flatter density profile and an enhanced progenitor mass-loss rate compared to those required to model the first peak. Our radio data alone cannot rule out the alternative scenario of an off-axis GRB powering the second radio peak, but the derived GRB parameters are somewhat unusual compared to typical values found for cosmological long GRBs. On the other hand, Chandra X-ray observations carried out during the second radio peak are compatible with the off-axis GRB hypothesis, within the large measurement errors. We conclude that VLBI measurements of the PTF11qcj radio ejecta are needed to unambiguously confirm or rule out the off-axis GRB jet scenario.
\end{abstract}

Key words: supernovae: general - supernovae: individual (PTF11qcj)

\section{Introduction}

The reason why some massive stars explode as supernovae $(\mathrm{SNe})$ and others as rare $\gamma$-ray bursts (GRBs) remains a mystery. Type $\mathrm{Ib} / \mathrm{c}$ supernovae $(\mathrm{SNe})$ are the result of the core collapse of massive stars, specifically the ones that have shed their hydrogen, and possibly helium, envelopes. Massive Wolf-Rayet stars and stars in close binary systems, that have completely lost their outer hydrogen layer due to stellar wind or through Roche-lobe overflow, respectively, are possible progenitors of these SNe (Ensman \& Woosley 1988; Gal-Yam 2017). Long duration ( $\gtrsim 2 s$ ) GRBs, with their engine-driven collimated outflows, are also thought to originate from the core collapse of massive stars (Paczyński 1998; Woosley \& Bloom 2006), being a rare subset of Type $\mathrm{Ib} / \mathrm{c}$ SNe. The leading scenario is that all long GRBs are accompanied by corecollapse $\mathrm{SNe}$ (though not all $\mathrm{Ib} / \mathrm{c} \mathrm{SNe}$ are accompanied by GRBs). In some long GRBs, the SN light may go undetected due to reasons such as large distances, poor localizations, dust extinction and galaxy contamination (Woosley \& Bloom 2006). It is noteworthy, however, that some nearby, well-localized GRBs without significant dust obscuration have been identified without an SN association (Fynbo et al. 2006; Gal-Yam et al. 2006).

So far, $\sim 11$ Type $\mathrm{Ib} / \mathrm{c}$ SNe have been discovered in connection with GRBs (Modjaz et al. 2016), pointing to a relationship between the two events. These include SN 1998bw (Galama et al. 1998) associated with GRB 980425, and a few more SNe (2003dh, 2003lw, 2006aj, 2010bh, 2010ma, 2012bz, 2013cq, 2013dx, 2013ez; Matheson et al. 2003; Stanek et al. 2003; Malesani et al. 2004; Pian et al. 2006; Sparre et al. 2011; Bufano et al. 2012; Xu et al. 2013; Cano et al. 2014; Schulze et al. 2014; D'Elia et al. 2015). SNe with a GRB association are generally more energetic than typical Type $\mathrm{Ib} / \mathrm{c} \mathrm{SNe}$ (Berger et al. 2003; Mazzali et al. 2003, 2006), with explosion kinetic energies of $\sim 10^{52} \mathrm{erg}$ (Mazzali et al. 2014) for the former, and $\sim 10^{51}$ erg (Taddia et al. 2015) for the latter, and also have broad features in their optical spectra (BL-Ic; Woosley \& Bloom 2006; Gal-Yam 2017) that imply high photospheric velocities.

In the radio, most "ordinary" BL-Ic SNe go undetected or are "radio-quiet" $\left(L_{\mathrm{GHz}} \lesssim 10^{26} \mathrm{erg} \mathrm{s}^{-1} \mathrm{~Hz}^{-1}\right.$; Berger et al. 2003; Soderberg et al. 2006; Corsi et al. 2016). On the other hand, the GRB-associated SN 1998bw was three orders of magnitude more radio luminous than, for example, the ordinary BL-Ic SN 2002ap, although a few orders of magnitude less radio luminous than cosmological GRB afterglows (see, e.g., Kulkarni et al. 1998; Chandra \& Frail 2012; Corsi et al. 2016, and references therein). Because radio emission probes the fastest ejecta, radio-loud BL-Ic SNe are more likely to be engine-driven (i.e., associated with GRBs). However, the radioloud Ib/c SN 2009bb showed no clear evidence for an association with a (high-luminosity) GRB (Soderberg et al. 2010; Pignata et al. 2011), opening the question of whether there is a class of core-collapse explosions with properties in 
between ordinary BL-Ic SNe and GRBs. In fact, the $\gamma$-ray energy of several GRBs with a spectroscopic SN association is also lower than that of typical cosmological GRBs (Amati et al. 2002; Mazzali et al. 2014). This suggests that low-luminosity GRBs themselves may represent a distinct population of intrinsically lower-energy events (Waxman 2004; Bromberg et al. 2011), although (for at least some of them) an interpretation as ordinary GRBs observed off-axis is also possible (Eichler \& Levinson 1999; Yamazaki et al. 2003).

Off-axis GRBs are a natural expectation of the fireball model (e.g., Rhoads 1997; Piran 2004). An off-axis GRB accompanying a relativistic (engine-driven) BL-Ic SN should become visible at late times in the radio (Perna \& Loeb 1998; Paczynski 2001; Waxman 2004), thus representing a potential source of radio-loud emission with characteristic timescales $(\gtrsim 100$ days since explosion) much longer than the radio peak time of 1998bw-like SNe (10-20 days since explosion; Kulkarni et al. 1998). The discovery of an off-axis long GRB associated with a BL-Ic SN remains, as of today, yet to be achieved. In fact, all previous claims of off-axis GRB discoveries (Granot \& Ramirez-Ruiz 2004; Paragi et al. 2010) have subsequently been ruled out (Bietenholz \& Bartel 2007; Soderberg et al. 2010; Bietenholz et al. 2014).

One of the challenges in searching for off-axis events is the fact that the characteristic late-time peaking radio light curve of an off-axis GRB (whose emission enters our line of sight after substantial deceleration has occurred) may, at first glance, look similar to that of a nonrelativistic SN whose ejecta are interacting strongly with a dense circumstellar medium (CSM). In radio SNe powered by synchrotron self-absorbed emission, the radio peak luminosity and the peak timescale probe the ejecta speed. Particularly, for a given radio luminosity, the later the peak time, the smaller the ejecta speed (Chevalier 1998; Berger et al. 2003). A relatively slow turn-on in the radio has been observed in radio-bright nonrelativistic Ib/c SNe such as SN 1979C and SN $1988 Z$ (Weiler et al. 1986; van Dyk et al. 1993), thought to be powered by shock interaction with a high-density medium (van Dyk et al. 1993). SN 2001em, SN 2003bg, SN 2004cc, SN 2004dk, SN 2004gq, and SN 2007bg (Wood-Vasey et al. 2003; Granot \& Ramirez-Ruiz 2004; Soderberg et al. 2006; Quimby et al. 2007; Wellons et al. 2012; Salas et al. 2013) are other more recent examples of (nonrelativistic) SNe that exhibit late-time radio emission arising from CSM density variations. In summary, distinguishing between CSM interaction and offaxis GRB jets requires an accurate analysis of broadband data sets.

Here, we present late-time radio observations of PTF11qcj, a bright BL-Ic SN for which the radio luminosity is $\sim 10^{29} \mathrm{erg} \mathrm{s}^{-1} \mathrm{~Hz}^{-1}$, comparable to that of SN 1998bw (see, e.g., Figure 1 of this paper; Corsi et al. 2014). Our extended radio follow-up observations of PTF11qcj show evidence for the presence of a second, late-time peak in its radio light curve. While the first radio peak pointed to a speed of the fastest ejecta of $\approx 0.3-0.5 c$ and a high progenitor mass-loss rate $\left(\sim 10^{-4} M_{\odot} \mathrm{yr}^{-1}\right)$ indicative of strong CSM interaction (Corsi et al. 2014), here we focus on the analysis of the second latetime radio peak within the two possible scenarios of strong CSM interaction and off-axis GRB. Our extensive radio data set is presented in Section 2; modeling of the radio data is discussed in Section 3; and results are presented in Section 4. In Section 5 we conclude.

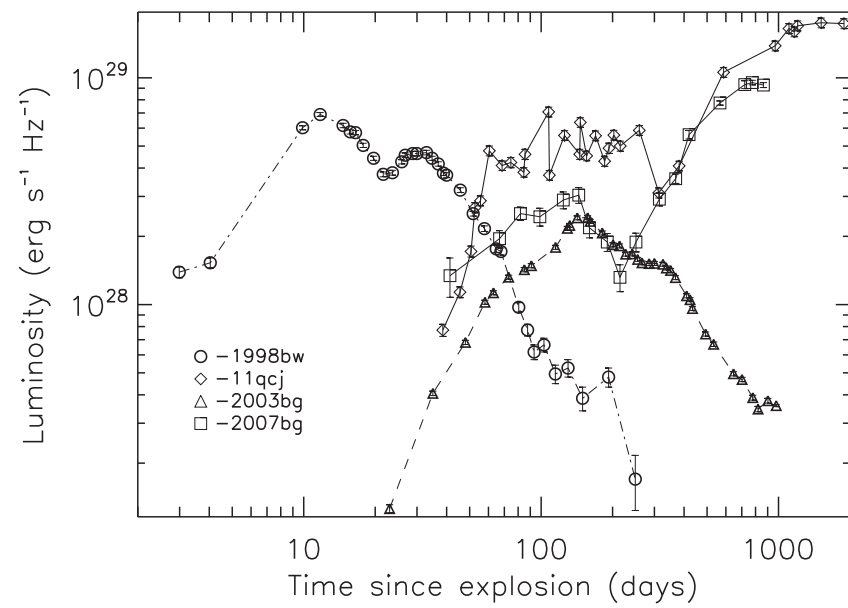

Figure 1. Radio luminosity of PTF11qcj compared to the GRB-associated SN $1998 \mathrm{bw}$ and other Ibc/Ilb supernovae that show late-time radio rebrightening (SN 2003bg and 2007bg; Soderberg et al. 2005; Salas et al. 2013). All data are at $5 \mathrm{GHz}$.

\section{Observations}

PTF11qcj was discovered by the Palomar Transient Factory (PTF; Rau et al. 2009) on 2011 November 1st UTC (MJD 55866), at $\alpha=13^{\mathrm{h}} 13^{\mathrm{m}} 41^{\mathrm{s}} .51, \delta=+47^{\circ} 17^{\prime \prime} 57^{\prime \prime} 0$, and at a redshift of $z=0.028$, corresponding to a luminosity distance of $d_{L} \approx 124 \mathrm{Mpc}$ (Corsi et al. 2014). Early optical observations and (radio-to-X-ray) follow-up data are presented in Corsi et al. (2014). In this section we describe later spectroscopic data and radio/X-ray follow-up observations.

\subsection{Spectroscopic Follow-up}

The location of PTF11qcj was observed with the Low Resolution Spectrograph-2 (LRS-2) attached to the HobbyEberly Telescope (HET) on 2017 February 22 UT. LRS-2 is a twin IFU spectrograph having two arms each: LRS2-B covers the range between 3700-4700 $\AA$ (blue arm) and 4600-7000 (orange arm) with resolving power of 1900 and 1100, respectively, while LRS2-R covers the range from 6500 to $8400 \AA$ (red arm) and from 8200 to $10500 \AA$ (far-red arm) at a resolution of 1800 in each arm (Chonis et al. 2014, 2016). Each IFU maps a $12^{\prime \prime} \times 6^{\prime \prime}$ area on the sky covered by 280 fibers. The coverage is complete, so no dithering is required.

We utilized the red arm of LRS2-R to collect spectra in the vicinity of the SN. Figure 2 shows the SDSS $r$-band frame of the host galaxy of PTF11qcj with the SN position marked in red. The blue rectangle indicates the position of the LRS2-R IFU (it was not completely centered on the $\mathrm{SN}$ due to a minor pointing issue). A spectrum at the $\mathrm{SN}$ position was extracted by median-combining the signal of the three closest fibers. Wavelength calibration was computed using $\mathrm{FeAr}$ spectral lamp observations. Flux calibration was performed by comparing the observed and flux-calibrated cataloged spectra of the standard star HD 84937.

The final LRS2 spectrum of PTF11qcj is plotted in Figure 3 together with the spectrum obtained with the Keck IIDEIMOS on 2012 March 20 (Corsi et al. 2014). This plot suggests that the broad SN features that were clearly visible in the Keck spectrum $5 \mathrm{yr}$ ago (typical nebular features due to forbidden transitions of neutral oxygen [O I] $\lambda \lambda 6300,6364$ and ionized [Ca II] $\lambda \lambda 7291,7324)$, may still be present in the 


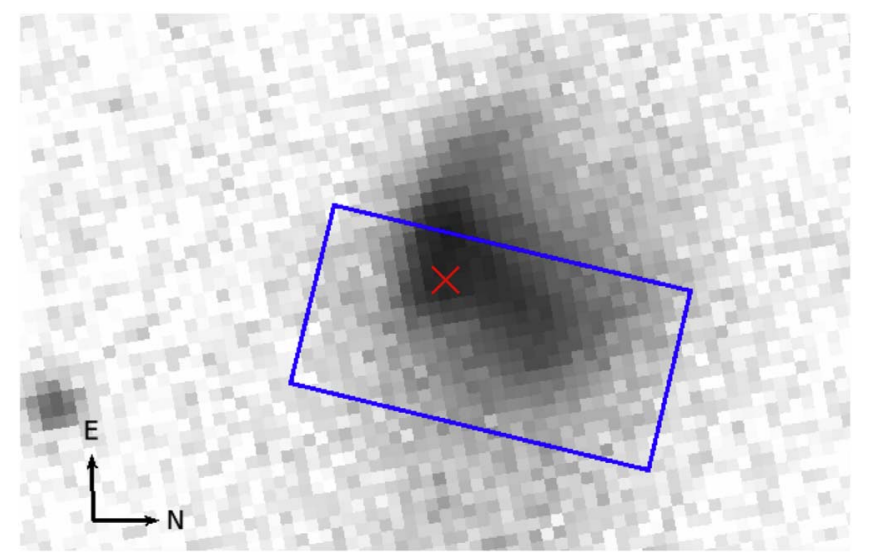

Figure 2. SDSS image of the host galaxy of PTF11qcj. The SN position is marked with the red cross. The position of the LRS2 IFU is indicated by the blue rectangle.

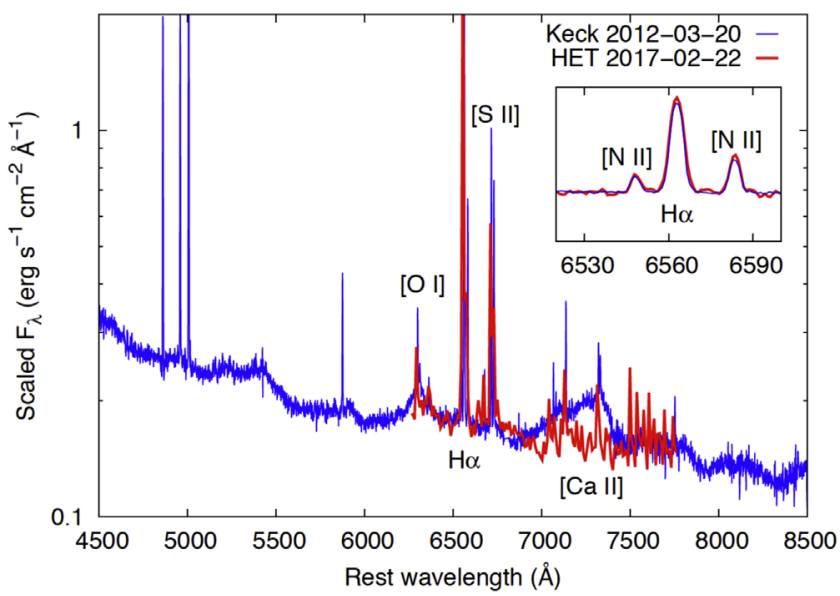

Figure 3. HET/LRS-2 spectrum with an exposure time of $2400 \mathrm{~s}$ from 2017 February 22 UT taken at the position of PTF11qcj (red curve) and the Keck DEIMOS spectrum from 2012 March 20 UT (blue curve). The inset zooms in on the narrow $\mathrm{H} \alpha$ lines visible in the recent HET-LRS2 spectrum, which are nearly identical to those in the older Keck spectrum, confirming that they are from the host.

new HET-LRS2 spectrum although with a smaller signal-tonoise. Also, the bright, narrow $\mathrm{H} \alpha$ feature present in both spectra appears basically unchanged (in both strength and width). No broad $\mathrm{H} \alpha$ feature can be identified in the HETLRS2 spectrum of PTF11qcj (as was the case for the older Keck spectrum during the first radio peak), thus excluding H-rich CSM interaction similar to SN 2014C (Milisavljevic et al. 2015). As discussed in what follows, interaction with an $\mathrm{H}$-poor CSM may explain the late-time radio rebrightening.

\subsection{Radio Follow-up}

The Very Large Array (VLA) follow-up observations presented here were carried out between 2014 June 1 UT (MJD 56809) and 2016 December 7 UT (MJD 57729). The data were taken at the nominal central frequencies of 2.5, 3.5, 7.4, 13.5, and $16 \mathrm{GHz}$ with a nominal bandwidth of $2 \mathrm{GHz}$. $3 \mathrm{C} 286$ and $\mathrm{J} 1327+4326$ were used as flux and phase calibrators, respectively.
The Common Astronomy Software applications (CASA; McMullin et al. 2007) ${ }^{11}$ was used to calibrate, flag, and image the data. The automated VLA calibration pipeline for CASA was used to calibrate the raw data. Images were formed from the visibility data using the CLEAN algorithm (Högbom 1974). The image size was set to $(1024 \times 1024)$ pixels, and the pixel size was determined as one-fourth of the nominal beamwidth. The images were cleaned using natural weighting for 10,000 iterations or until a threshold of $\sim 0.03 \mathrm{mJy}(\sim 3 \sigma)$ was reached. The source flux at each epoch was calculated using imstat i.e., as the flux corresponding to the brightest pixel within a circle centered around the PTF position with a radius of $2^{\prime \prime}$ (comparable to the typical $R$-band seeing of PTF images; Law et al. 2009). Flux errors are calculated as the quadratic sum of the rms map error and a 5\% fractional error that accounts for errors in the flux calibration (Weiler et al. 1986; Corsi et al. 2014). We have verified that peak fluxes estimated using imstat are consistent within errors to peak fluxes and total fluxes estimated by using imfit, which fits a two-dimensional Gaussian component to the source. We also note that results from imfit do not show evidence for extended emission, confirming that the radio counterpart of PTF11qcj is a point source up to $\approx 0$ !. 27 , which is the beam size in the VLA A array configuration at $7.4 \mathrm{GHz}$.

Figure 4 shows the radio light curves of PTF11qcj, with the new data from MJD 56809 onward plotted along with the data from Corsi et al. (2014). The fluxes at each MJD and frequency for the latest observations are reported in Table 1 .

\subsection{X-Ray Follow-up}

The location of PTF11qcj was imaged by the Advanced CCD Imaging Spectrometer (Garmire et al. 2003) on board Chandra on 2018 February 23 UT and 2018 February 26 UT. A total exposure time of $40 \mathrm{ks}$ was obtained with the location of PTF11qcj positioned at the nominal aimpoint of chip S3 (i.e., ACIS-S). We detect a faint unresolved source at this position, with a $0.5-7.0 \mathrm{keV}$ count rate of $(1.6 \pm 0.3) \times 10^{-3} \mathrm{ct} \mathrm{s}^{-1}$ (corrected for background and the instrument PSF using the task srcflux from the Chandra Interactive Analysis of Observations package v4.9; $90 \%$ confidence). Assuming a power-law spectrum with a photon index of $\Gamma \approx 1.7$ and a Galactic $N_{\mathrm{H}}$ column density of $\approx 10^{20} \mathrm{~cm}^{-2}$ (Corsi et al. 2014), this corresponds to an unabsorbed $0.3-8.0 \mathrm{keV}$ flux of $(2.2 \pm 0.4) \times 10^{-14} \mathrm{erg} \mathrm{cm}^{-2} \mathrm{~s}^{-1}$ (which can be directly compared to the flux measurements obtained during previous epochs and reported in Corsi et al. 2014).

\section{Radio Modeling}

As shown in Figure 4, the higher-frequency (13.5 and 16 $\mathrm{GHz}$ ) radio light curves of PTF11qcj appear double-peaked. At lower frequencies, a late-time rebrightening is also evident. We thus identify two phases in the evolution of the radio emission, with the first peak in between $\sim$ MJD 55842 and $\sim$ MJD 56101, and the second starting from $\sim$ MJD 56101. We remind the reader that, as noted in Corsi et al. (2014), the abrupt flux variations observed during the first peak around 100 days since explosion result from data taken when the VLA antennas were switching between configurations, and may be affected by calibration issues.

\footnotetext{
${ }^{11}$ Available online at http://casa.nrao.edu.
} 

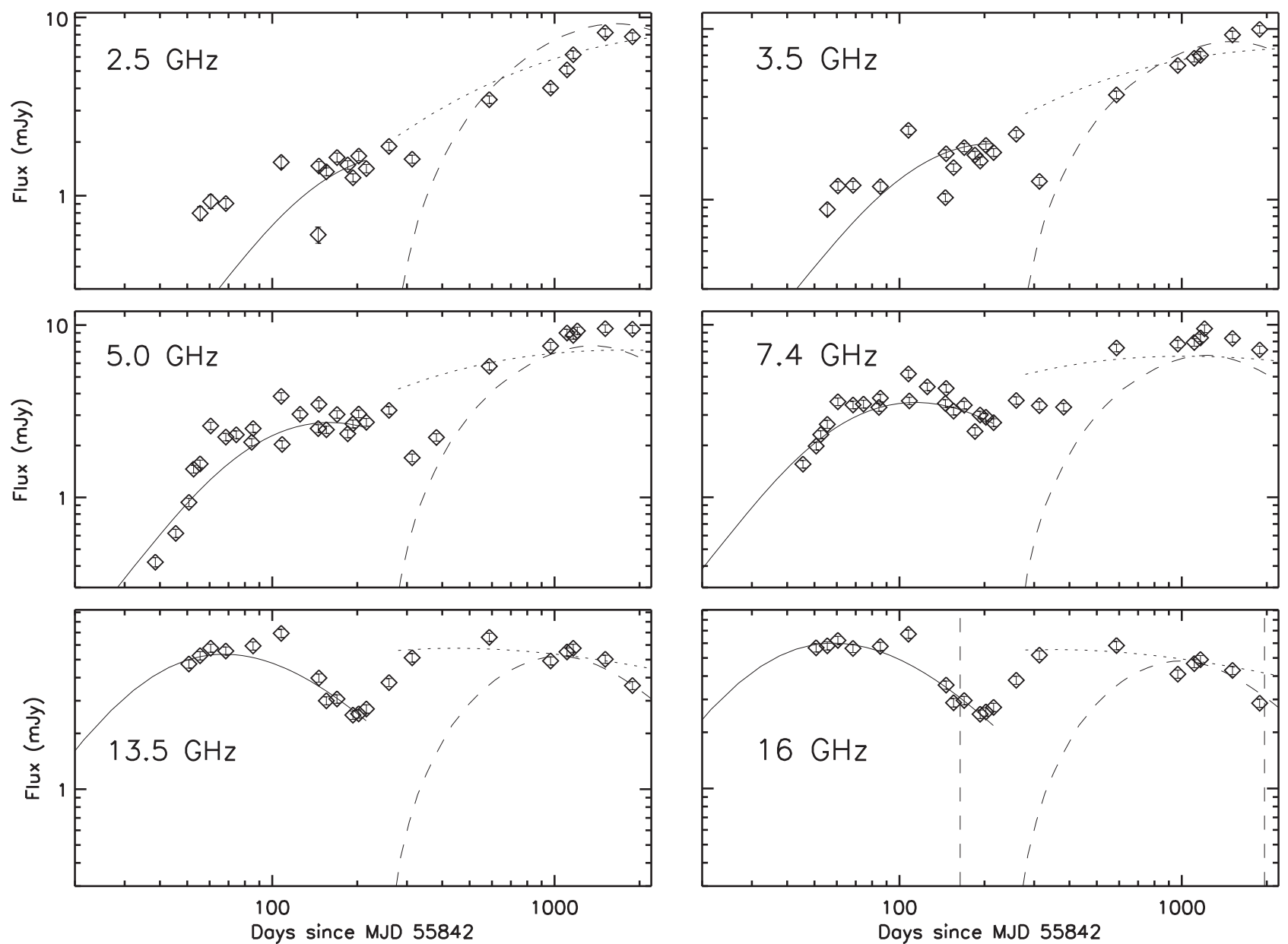

Figure 4. Radio light curves of PTF11qcj obtained with the VLA at six different frequencies. The first radio peak is modeled within a standard SSA model as described in Corsi et al. (2014; Model 0 in Table 2; solid curves). The rebrightening phase is modeled in two different scenarios: (i) within the standard SSA model varying $B_{0}, s$, and $\xi$ (dotted curves; Model 1); and (ii) within an off-axis afterglow model with a constant density ISM and varying $\theta_{0}, E_{\text {iso }}, n_{\text {ISM }}$, and $\theta_{\text {obs }}$ (dashed curves). The vertical dashed lines in the bottom right panel mark the epochs of the last Keck (Corsi et al. 2014) and HET (Figure 3) spectra on MJD 56006 and 57806 respectively.

As discussed in Section 1, bright radio emission in a SN may be powered by the presence of a central engine, or via the interaction of the ejecta with the the CSM. Within the CSMinteraction scenario, a double-peaked radio light curve may be attributed to density variations in the CSM (Soderberg et al. 2006) due to variable (potentially eruptive) mass loss from the $\mathrm{SN}$ progenitor, or clumping due to turbulence in the medium and violent outbursts (Wellons et al. 2012; Salas et al. 2013). In the case of a binary SN progenitor, variable radio emission may occur due to two different wind components from the two stars in the binary and/or the interaction of the shock with the common envelop or the wind termination shock (see, e.g., Wellons et al. 2012). On the other hand, in the engine-driven scenario, a late-time peak in the radio light curve may be expected from an off-axis GRB: as the ultra-relativistic jet transitions to the subrelativistic regime, it also spreads sideways resulting in a rebrightening of the radio SN light curve (e.g., Waxman 2004).

In light of the above, in what follows we model the PTF11qcj light curves within both the synchrotron self-absorption (SSA) model with CSM density variations (Soderberg et al. 2005), and the off-axis GRB afterglow model (van Eerten et al. 2010, 2012). We stress that the formulation of the SSA model used here is the simplest used in the literature. As already noted in Corsi et al. (2014), alternative scenarios invoking internal free-free absorption, or free-free absorption from a clumpy CSM, or a radial gradient in the electron temperature may also explain and/or contribute to the attenuation mechanisms during the earlier part of the first peak (e.g., Weiler et al. 1990; Chevalier 1998; Fransson \& Björnsson 1998).

\subsection{CSM-interaction SSA Model}

The model in Soderberg et al. (2005) describes the radio emission from SN ejecta interacting with the CSM. The radio flux density at time $t$ and frequency $\nu$ is given by:

$$
\begin{aligned}
F(t, \nu)= & C_{f}\left(\frac{t-t_{e}}{t_{0}}\right)^{\left(4 \alpha_{r}-\alpha_{B}\right) / 2} \\
& \times\left[1-\exp ^{-\tau_{\nu}^{\xi}(t)}\right]^{1 / \xi} \nu^{5 / 2} F_{3}(x) F_{2}^{-1}(x),
\end{aligned}
$$

with the optical depth given by

$$
\tau_{\nu}(t)=C_{\tau}\left(\frac{t-t_{e}}{t_{0}}\right)^{(3+p / 2) \alpha_{B}+(2 p-3) \alpha_{r}-2(p-2)},
$$


Table 1

Summary of the Late-time VLA Observations of PTF11qcj

\begin{tabular}{|c|c|c|c|c|}
\hline $\begin{array}{l}\text { Start Time } \\
\text { (MJD) }\end{array}$ & $\begin{array}{l}\text { Epoch } \\
\text { (days) }\end{array}$ & Observatory & $\begin{array}{l}\text { Freq. } \\
(\mathrm{GHz})\end{array}$ & $\begin{array}{l}\text { Flux } \\
(\mathrm{mJy})\end{array}$ \\
\hline 56809.993 & 967 & VLA:A & 2.5 & $4.01 \pm 0.20$ \\
\hline$" 1$ & " & VLA:A & 3.5 & $6.10 \pm 0.30$ \\
\hline$"$ & " & VLA:A & 5.0 & $7.55 \pm 0.37$ \\
\hline " & " & VLA:A & 7.4 & $7.73 \pm 0.38$ \\
\hline " & " & VLA:A & 13 & $4.92 \pm 0.24$ \\
\hline " & " & VLA:A & 16 & $4.09 \pm 0.20$ \\
\hline 56948.910 & 1106 & VLA:C & 3.5 & $6.74 \pm 0.33$ \\
\hline$"$ & " & VLA:C & 5.0 & $9.00 \pm 0.45$ \\
\hline " & " & VLA:C & 7.4 & $7.90 \pm 0.39$ \\
\hline " & " & VLA:C & 13 & $5.50 \pm 0.27$ \\
\hline " & " & VLA:C & 16 & $4.66 \pm 0.23$ \\
\hline 57006.658 & 1164 & VLA:C & 2.5 & $6.18 \pm 0.30$ \\
\hline " & " & VLA:C & 3.5 & $7.00 \pm 0.35$ \\
\hline " & " & VLA:C & 5.0 & $8.71 \pm 0.43$ \\
\hline " & " & VLA:C & 7.4 & $8.39 \pm 0.41$ \\
\hline " & " & VLA:C & 13 & $5.76 \pm 0.28$ \\
\hline " & " & VLA:C & 16 & $4.90 \pm 0.24$ \\
\hline 57046.262 & 1204 & VLA:CnB & 5.0 & $9.25 \pm 0.46$ \\
\hline " & " & VLA:CnB & 7.4 & $9.49 \pm 0.47$ \\
\hline 57354.492 & 1512 & VLA:D & 13 & $5.02 \pm 0.25$ \\
\hline " & " & VLA:D & 16 & $4.29 \pm 0.21$ \\
\hline " & " & VLA:D & 5.0 & $9.54 \pm 0.47$ \\
\hline " & " & VLA:D & 7.3 & $8.33 \pm 0.41$ \\
\hline " & " & VLA:D & 2.4 & $8.21 \pm 0.41$ \\
\hline " & " & VLA:D & 3.4 & $9.23 \pm 0.46$ \\
\hline 57729.458 & 1887 & VLA:A & 13 & $3.62 \pm 0.18$ \\
\hline$"$ & " & VLA:A & 16 & $2.86 \pm 0.14$ \\
\hline " & " & VLA:A & 5.0 & $9.46 \pm 0.47$ \\
\hline$"$ & " & VLA:A & 7.3 & $7.15 \pm 0.35$ \\
\hline$"$ & " & VLA:A & 2.4 & $7.80 \pm 0.39$ \\
\hline$" 1$ & " & VLA:A & 3.4 & $9.94 \pm 0.49$ \\
\hline
\end{tabular}

Note. From left to right: observation start time (MJD), epoch in days since the estimated explosion date (MJD 55842; see Corsi et al. 2014), array configuration, central frequency, and peak flux.

where $C_{f}, C_{\tau}$ are normalization constants, $F_{2}, F_{1}$ are Bessel functions, and $x=2 / 3\left(\nu / \nu_{m}\right)$ where $\nu_{m}$ is the critical synchrotron frequency, $t_{0}$ is a reference epoch, $t_{e}$ is the explosion time, $p$ is the electron energy index, and $\xi=[0,1]$ describes the sharpness of the spectral break between optically thin and thick regimes (Soderberg et al. 2005). In the above equations, $\alpha_{r}$ and $\alpha_{B}$ are the temporal indices of the shock radius $r$ and the magnetic field $B$, respectively, such that

$$
r=r_{0}\left(\frac{t-t_{e}}{t_{0}}\right)^{\alpha_{r}}
$$

and

$$
B=B_{0}\left(\frac{t-t_{e}}{t_{0}}\right)^{\alpha_{B}},
$$

where $r_{0}$ and $B_{0}$ are the radius and magnetic field at the reference epoch $t_{0}$. The expansion of the $\mathrm{SN}$ shock is described by:

$$
\alpha_{r}=\frac{n-3}{n-s},
$$

where $\rho_{\mathrm{ej}} \propto r^{-n}$ is the density profile of the outer SN ejecta, and $\rho_{\mathrm{CSM}} \propto n_{e} \propto r^{-s}$ is that of the shocked CSM (or shocked electron density). The self-similar conditions $s<3$ and $n>5$ (Chevalier 1982) result in $\alpha_{r}<1$.

In the standard scenario, the magnetic energy density and the relativistic electron energy density are constant fractions, $\epsilon_{B}$ and $\epsilon_{e}$, respectively, of the post-shock energy density. Under these assumptions:

$$
\alpha_{B}=\frac{(2-s)}{2} \alpha_{r}-1,
$$

and the minimum Lorentz gamma factor and the critical synchrotron frequency of the radiating electrons read:

$$
\begin{gathered}
\gamma_{m}=\gamma_{0}\left(\frac{t-t_{e}}{t_{0}}\right)^{2\left(\alpha_{r}-1\right)}, \\
\nu_{m}=\nu_{m, 0}\left(\frac{t-t_{e}}{t_{0}}\right)^{\left(10 \alpha_{r}-s \alpha_{r}-10\right) / 2} .
\end{gathered}
$$

The electron number density within the shocked CSM is given by:

$$
n_{e}=\frac{p-2}{p-1} \frac{\left(\epsilon_{e} / \epsilon_{B}\right) B_{0}^{2}}{8 \pi m_{e} c^{2} \gamma_{m, 0}}\left(\frac{t-t_{e}}{t_{0}}\right)^{-s \alpha_{r}} \mathrm{~cm}^{-3},
$$

where $m_{e}$ is the electron mass, and $c$ is the speed of light. The SN progenitor mass-loss rate reads:

$$
\dot{M}=\frac{8 \pi n_{e} m_{p} r_{0}^{2} v_{w}}{\eta}\left(\frac{t-t_{e}}{t_{0}}\right)^{\alpha_{r}(2-s)},
$$

where $m_{p}$ is the proton mass and $v_{w}$ is the wind velocity, while $\eta$ describes the thickness, $r / \eta$, of the radiating shell at radius $r$. Finally, the ejecta energy reads:

$$
E=\frac{4 \pi r_{0}^{2} B_{0}^{2}}{\eta 8 \pi \epsilon_{B}}\left(\frac{t-t_{e}}{t_{0}}\right)^{5 \alpha_{r}-s \alpha_{r}-2} .
$$

Hereafter, we work under the equipartition hypothesis and set $\epsilon_{e}=\epsilon_{B}=0.33$. We note that departures from equipartition would imply $\epsilon_{e} / \epsilon_{B} \gtrsim 1$ and likely point to $\epsilon_{B} \lesssim 0.33$, thus increasing both the shocked electron number density (and, in turn, the estimated mass-loss rate; see Equations (9)-(10)), and the energy budget (Equation (11)).

As is evident from Equations (1)-(2) and (4)-(8), the observed flux at a given frequency $\nu$ and time $t$ depends on $C_{f}$, $C_{\tau}, p, s, \alpha_{r}, \nu_{m, 0}, \xi$, and $t_{e}$. Since $C_{f}, C_{\tau}$ can be expressed in terms of $r_{0}$ and $B_{0}$ (see Equations (6)-(8) in Soderberg et al. 2005), the observed flux ultimately depends on $r_{0}, B_{0}, p, s, \alpha_{r}$, $\nu_{m, 0}, \xi$, and $t_{e}$, which we determine by comparison with the observed data using a $\chi^{2}$ minimization procedure (see Corsi et al. 2014). In modeling the radio emission from PTF11qcj, following Corsi et al. (2014), we set $t_{0}=10$ days and $\nu_{m, 0} \approx 1 \mathrm{GHz}$.

\subsection{Off-axis GRB Afterglow Model}

In a scenario in which PTF11qcj is powered by a central engine, we may interpret its double-peaked radio light curves 
Table 2

Best-fit Parameters for the Standard SSA Model Described in Section 3.1

\begin{tabular}{lcc}
\hline \hline Parameter & Model 0 & Model 1 \\
\hline$r_{0}(\mathrm{~cm})$ & $1.0 \times 10^{16}$ & $1.0 \times 10^{16}$ \\
$\xi$ & 0.24 & 0.19 \\
$\alpha_{r}$ & 0.81 & 0.81 \\
$t_{e}$ & 55842 & 55842 \\
$s$ & 2.0 & 1.13 \\
$B_{0}(\mathrm{G})$ & 6.5 & 3.2 \\
$p$ & 3.0 & 3.0 \\
\hline$\alpha_{B}$ & -1.0 & -0.64 \\
$\gamma_{m, 0}$ & 7.4 & 11 \\
$\alpha_{\gamma}\left(\mathrm{cm}^{-3}\right)$ & -0.38 & -0.38 \\
$n_{e, 0}$ & $2.3 \times 10^{4}$ \\
$\alpha_{n_{e}}$ & $1.4 \times 10^{5}$ & -0.91 \\
$\dot{M}_{0}\left(M_{\odot} \mathrm{yr}^{-1}\right)$ & -1.6 & $1.7 \times 10^{-5}$ \\
$\alpha_{\dot{M}}$ & $9.8 \times 10^{-5}$ & 0.70 \\
$E_{k, 0}(\mathrm{erg})$ & 0.0 & $1.7 \times 10^{48}$ \\
$\alpha_{E_{k}}$ & $7.1 \times 10^{48}$ & 1.1 \\
$\chi^{2} / \mathrm{dof}$ & 0.42 & $575 / 35$ \\
\hline
\end{tabular}

Note. Model 0 is the best fit for the first peak in the radio light curves, with fixed $p=3, s=2$, and $t_{e}=55842$. Model 1 is the best fit for the late-time rebrightening phase. For Model $1, B_{0}, s$, and $\xi$ are varied.

as a combination of radio emission from an uncollimated (nonrelativistic) SN shock interacting with a dense CSM (as described in Corsi et al. 2014), and that of an off-axis relativistic jet entering our line of sight at late times, as the jet decelerates and spreads sideways (Waxman 2004). The jet dynamics in the relativistic regime is described by the Blandford-Mckee solution (Blandford \& McKee 1976), and in the late nonrelativistic regime by the Sedov-von NeumannTaylor solution (Taylor 1946).

Because analytical solutions for the dynamics of a spreading and decelerating relativistic jet cannot fully capture the details (sideways expansion and transition to the nonrelativistic regime) of the blast wave evolution, hereafter we use the high-resolution relativistic hydrodynamic simulations by Zhang \& MacFadyen (2009) and van Eerten et al. (2012) for a jet expanding in a constant density ISM. These twodimensional simulations include the transition from the relativistic to nonrelativistic regime, which is essential to accurately model the GRB outflow at late times (Zhang \& MacFadyen 2009). The observed flux can be modeled as a function of eight parameters: the isotropic equivalent kinetic energy of the explosion, $E_{\text {iso }}$; the circumburst medium number density, $n_{\mathrm{ISM}}$; the jet half-opening angle, $\theta_{0}$; the observer's angle, $\theta_{\text {obs }}$; the fraction of internal energy in the shock going into magnetic fields, $\epsilon_{B}$; the fraction of internal energy going into accelerating electrons, $\epsilon_{e}$; the fraction of electrons shockaccelerated in a power-law energy distribution, $\xi_{N} \sim 1$; and the power-law index of the accelerated electron energy distribution, $p$. These parameters are determined by comparison with the observed fluxes (at each observed frequency and time) using a $\chi^{2}$ minimization procedure (van Eerten et al. 2012).

\section{Results}

\subsection{CSM-interaction Radio Fits}

In Table 2 and Figure 4 we report fit results for the second radio peak of PTF11qcj within the synchrotron SSA scenario described in Section 3.1 (Model 1). We impose a smooth radial evolution of the $\mathrm{SN}$ shock, i.e., we require that $r_{0}$ and $\alpha_{r}$ remain unchanged with respect to what was found during the first radio peak (Model 0), and attempt to model the second radio peak by varying the wind density profile ( $s$ and, in turn, $\alpha_{B}$; see Equation (5)) and the magnetic field $\left(B_{0}\right)$. This is justified by the consideration that a change in these parameters at fixed $r_{0}$ and $\alpha_{r}$ effectively corresponds to a change in CSM density as $F_{\nu} \propto B^{4} \propto n_{e}^{2}$ (Wellons et al. 2012). We also allow for variations in $\xi$. The model was fit to the data obtained after MJD 56429, which is around the time rebrightening occurs, yielding a $\chi^{2} /$ dof $=575 / 35$ (see Table 2 ). We note that including the rising data points in the fit results in $B_{0} \approx 1.9$, $s \approx 0.87$ and $\xi \approx 0.17$, and a $\chi^{2} /$ dof $=1703 / 49$.

The physical parameters derived from Model 1 best-fit results are shown in Figure 5. When deriving the mass-loss rate and energy implied by the best-fit results (from Equations (10)-(11)), we assume a shell thickness of $\eta=10$. Previous studies have assumed shell thicknesses in between $\eta=4$ (e.g., Soderberg et al. 2006; Wellons et al. 2012) and $\eta=10$ (Li \& Chevalier 1999; Frail et al. 2000; Soderberg et al. 2005). This range is consistent with shell widths of $\eta \approx 3-10$ measured via VLBI observations (Chevalier 1982; Bietenholz et al. 2003; Bartel et al. 2007; Roy et al. 2009). As is evident from Figure 5, the best fit (Model 1 in Table 2) requires an increase in energy and massloss rate at the start of the second radio peak due to the flattening of the density profile (i.e., smaller value of $s$ in Model 1 than in Model 0; see the top left and bottom right panels of Figure 5). This is similar to what was observed in, e.g., the CSM-interacting SN 2003bg (Soderberg et al. 2006).

In Figure 6 we show the uncertainties in the best-fit results for $s$ and $B_{0}$, which shows that during the second radio peak a flattening in the CSM profile is also required (compared to the first peak). We note that a flattening in the density profile may be attributed to passage through a termination shock (e.g., Chevalier et al. 2004), although the simplified analytical model used here does not allow us to properly account for other sources of possible density profile variations such as, e.g., clumpiness in the stellar wind. More generally, the fact that the best-fit model to the second peak favors a value of $s$ that is different from the standard $s=2$ supports the hypothesis of a time-varying mass loss.

\subsection{Off-axis GRB Radio Fits}

We use the off-axis afterglow model described in Section 3.2 to model the second radio peak in the PTF11qcj light curve. In our fits, $\epsilon_{e}=\epsilon_{B}=0.33$, and $p$ are held fixed. For a fireball expanding in a constant density medium, we find a best fit with an explosion energy of $E_{\text {iso }} \approx 7 \times 10^{52} \mathrm{erg}, \theta_{0} \approx 0.3 \mathrm{rad}$, ISM density $n_{\mathrm{ISM}} \approx 3 \times 10^{-5} \mathrm{~cm}^{-3}, \theta_{\mathrm{obs}} \approx 0.6 \mathrm{rad}$, and a $\chi^{2} / \mathrm{dof}=$ 1167/36 (Figure 4, dashed curve). The ISM density predicted by this model is rather low compared to typical long GRBs, which have $n_{\text {ISM }} \gtrsim 1 \mathrm{~cm}^{-3}$. However, low ISM densities although peculiar are not unseen in long GRB broadband afterglow modeling (Kumar \& Panaitescu 2000; Laskar et al. 2014, 2015; Alexander et al. 2017). We note that setting $n_{\mathrm{ISM}}=$ $1 \mathrm{~cm}^{-3}$ (as typical for long GRBs) and allowing $\theta_{0}$ and $\theta_{\text {obs }}$ to vary, returns a best fit with $E_{\mathrm{iso}} \approx 2 \times 10^{53} \mathrm{erg}, \theta_{0} \approx 0.05 \mathrm{rad}$, $\theta_{\text {obs }} \approx 0.17 \mathrm{rad}$, and a $\chi^{2} /$ dof $=4154 / 37$. 

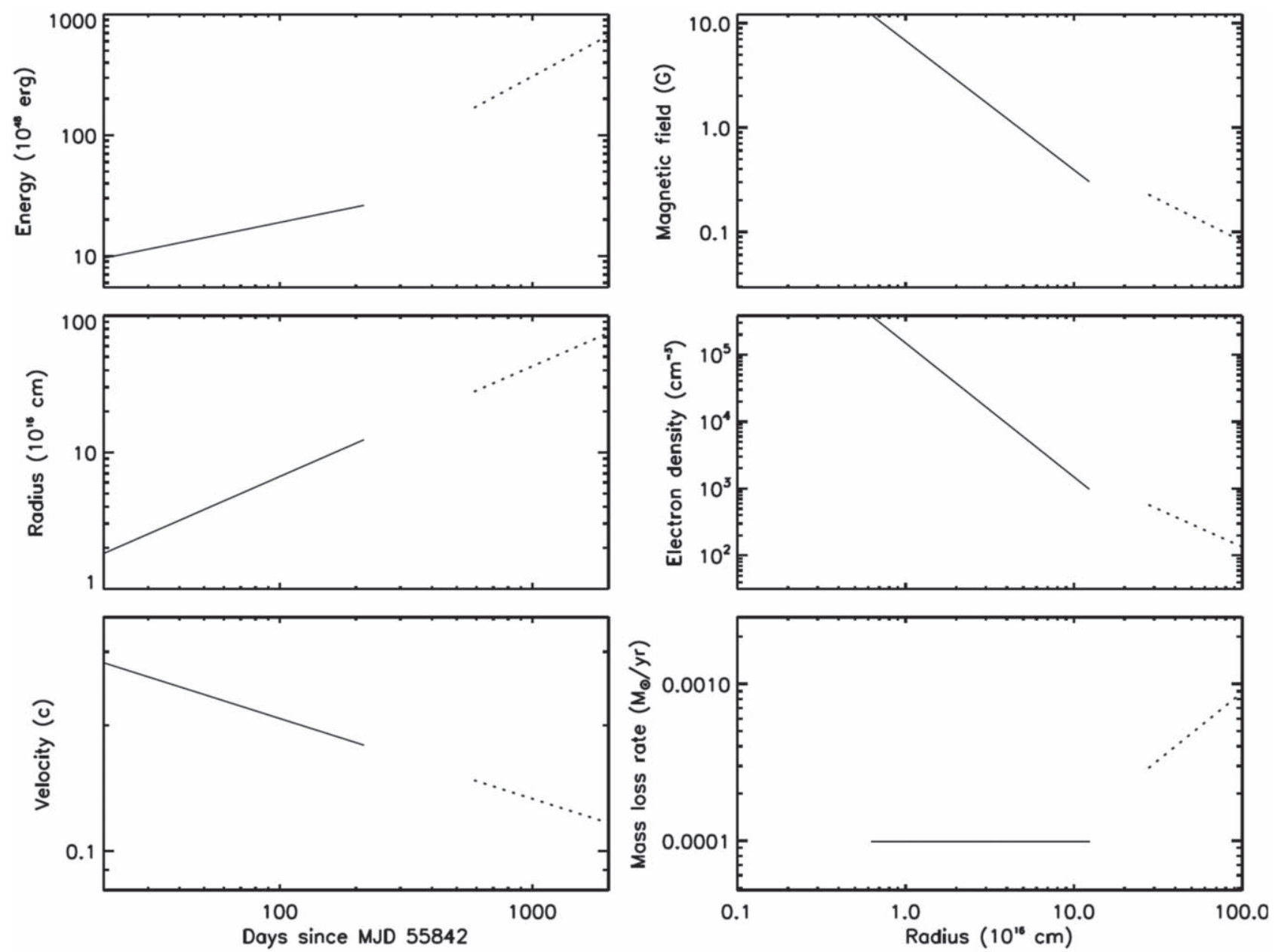

Figure 5. Physical parameters derived from Model 0 for phase 1 (solid curves), and from Model 1 (dotted curve) for phase two (rebrightening) of the radio light curve. Left column, from top to bottom: energy, radius, and velocity as a function of time. Right column, from top to bottom: radial profiles of the magnetic field, electron density, and mass-loss rate. The best-fit models are listed in Table 2.

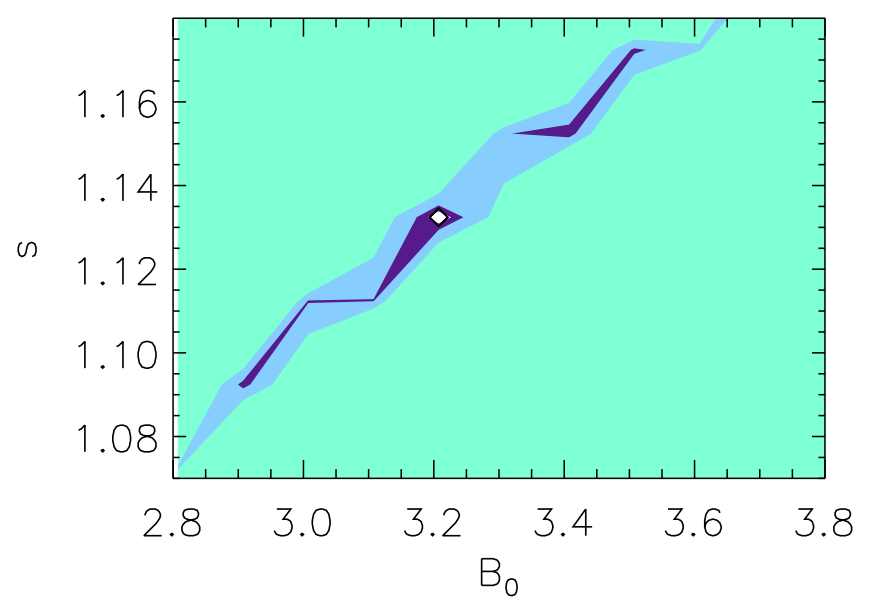

Figure 6. Best-fit results (diamonds) and confidence intervals for two interesting parameters for Model 1. The white, purple, light blue, and aqua green regions correspond to confidence intervals of $\lesssim 68 \%$, between $68 \%$ and $90 \%$, between $90 \%$ and $99 \%$, and $\gtrsim 99 \%$, respectively. See Table 2 for more details.

\subsection{Comparing Model Predictions and Data in the X-Rays}

Our past (Corsi et al. 2014) and recent observations of PTF11qcj with Chandra all yielded detections. Here, we compare our X-ray data set with the predictions from the
CSM-interacting SN and off-axis GRB scenarios described in Sections 4.1 and 4.2 .

For each of our X-ray observations, we conservatively calculate the implied $1 \mathrm{keV}$ flux density taking into account errors on both the X-ray spectral index (as estimated in Corsi et al. 2014), and on the measured count rate. More specifically, at each epoch we calculate the implied $1 \mathrm{keV}$ flux range by extrapolating the full $\pm 1 \sigma$ range on the measured count rate using a power law of index spanning the range $\Gamma=1.6_{-0.71}^{+0.81}$ (Corsi et al. 2014). This yields the $1 \mathrm{keV}$ fluxes (dots) and the large error bars shown in Figure 7. We then compare these extrapolated data points with specific model predictions.

Within the SSA model for CSM-interacting SNe, we expect the radio-to-X-ray spectral index to be $\beta \lesssim-(p-1) / 2$, and thus $\beta \lesssim-1$ for $p \approx 3$ (see Table 2). In Figure 7 we plot the SSA models shown in the bottom right panel of Figure 4, extrapolated to $1 \mathrm{keV}$ by using $\beta=-1$. As is evident from this figure, and as already noted by Corsi et al. (2014), the SSA model for the first radio peak (black solid line in Figure 4) can explain the observed X-ray emission if no spectral break is present between the radio and $\mathrm{X}$-rays (black solid line in Figure 7).

On the other hand, our latest Chandra data implies an $\mathrm{X}$-ray flux that falls above the extrapolation to $1 \mathrm{keV}$ of the best-fit SSA model for the second radio peak (dotted lines in 


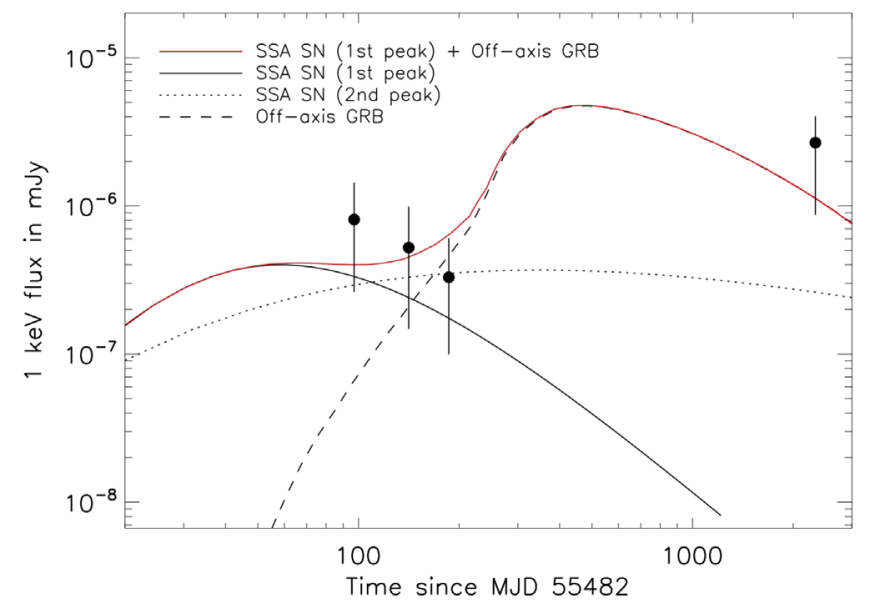

Figure 7. Chandra data of PTF11qcj (dots) are plotted together with the SSA models shown in the bottom right panel of Figure 4, extrapolated to $1 \mathrm{keV}$ by using $\beta=-1$. The SSA model for the first radio peak (black solid line) can explain the observed X-ray emission if no spectral break is present between the radio and X-rays. On the other hand, our latest Chandra data implies an X-ray flux in excess to what would be expected by extrapolating to $1 \mathrm{keV}$ the best-fit SSA model for the second radio peak (dotted line). The $1 \mathrm{keV}$ flux prediction from the off-axis GRB model discussed in Section 4.2 (dashed line) is marginally consistent with our last Chandra data point.

Figures 4 and 7). The $1 \mathrm{keV}$ flux predictions from the off-axis GRB model discussed in Section 4.2 (dashed lines in Figures 4 and 7) are instead consistent with this data point. Given the large measurement errors, as we discuss in the next section, VLBI observations are likely needed to securely confirm (or rule out) the off-axis GRB hypothesis.

\section{Discussion and Conclusion}

We have presented PTF11qcj late-time VLA observations up to $\sim 5 \mathrm{yr}$ since optical discovery, late-time spectroscopic follow-up with the HET at $\approx 5 \mathrm{yr}$ post-explosion, and late-time $\mathrm{X}$-ray follow-up with Chandra at $\sim 6 \mathrm{yr}$ since explosion. The radio luminosity of PTF11qcj is as high as that of the GRBassociated SN 1998bw. The radio light curves show a doublepeak profile, with the first peak emerging at $\approx 100$ days since explosion, and the second at $\approx 2000$ days $(\approx 5.5 \mathrm{yr})$ since explosion. We model the second radio peak (i) with CSM density variations in the standard synchrotron SSA model (Soderberg et al. 2005), and (ii) within an off-axis GRB model (van Eerten et al. 2012).

We find that density enhancements alone (Model 1) may explain the late-time rebrightening of PTF11qcj. Radio modeling suggests an enhanced mass-loss rate during the second radio peak. Even though precursor eruptions have mostly been detected in type IIn SNe, evidence for pre-SN activity was detected in the pre-explosion images of PTF11qcj around 2009 May-July (Corsi et al. 2014) hinting at the possibility of such mass-loss episodes being responsible for the enhanced mass-loss rate during the second peak. Assuming the explosion took place on MJD 55842, and with $r_{0} \sim$ $10^{16} \mathrm{~cm}$ and $\alpha_{r} \approx 0.8$, the shock radius would have reached $r \approx 4 \times 10^{17} \mathrm{~cm}$ around 1000 days since the explosion (i.e., around the peak of the rebrightening phase). If material from the pre-SN activity observed in 2009 ( $~ 860$ days before the explosion) was responsible for the radio rebrightening, the progenitor wind would have traveled at a speed $\sim 24,000 \mathrm{~km} \mathrm{~s}^{-1}$, which is much larger than typical stellar wind speeds of strippedenvelope core-collapse SN progenitors $\left(\sim 1000 \mathrm{~km} \mathrm{~s}^{-1}\right)$. The nondetection of H-rich material (to a level distinguishable by our HET/LRS-2 spectrum) during the second radio peak also implies that H-rich layers would have been shed well before the 2009 pre-SN activity.

If an off-axis GRB is invoked to explain the late-time radio rebrightening, a very low $n_{\text {ISM }}$ value is required to fit the data. This off-axis model is also marginally compatible with the flux measured in X-rays during our latest Chandra observation of PTF11qcj.

Mazzali et al. (2014) pointed out that in GRB-SNe, the SN carries most of the energy compared to the $\gamma$-ray energy of the jet - an indication that the $\mathrm{SN}$ is powered by a central engine. Since we cannot rule out the off-axis GRB scenario for PTF11qcj, we attempt to estimate the $\gamma$-ray energy of a hypothetical GRB associated with PTF11qcj under the assumption that this was an engine-driven SN. From the analysis of four GRB-SNe, Li (2006) finds that the peak spectral energy of GRBs and the peak bolometric luminosity of the underlying supernova are related by $\nu_{\gamma, \text { peak }}=90.2 \mathrm{keV}\left(L_{\mathrm{SN} \text {,peak }} / 10^{43} \mathrm{erg} \mathrm{s}^{-1}\right)^{4.97}$. Considering the peak bolometric luminosity of PTF11qcj $\gtrsim 10^{9.3} L_{\odot}$ (Corsi et al. 2014), the peak $\gamma$-ray energy of a hypothetical GRB can be expected to be $\gtrsim 23 \mathrm{keV}$. Then assuming the correlation between the $\nu_{\gamma \text {,peak }}$ and $E_{\text {iso }}$ as $\nu_{\gamma \text {,peak }}=97 \mathrm{keV}\left(\mathrm{E}_{\gamma, \text { iso }} / 10^{52} \mathrm{erg} \mathrm{s}^{-1}\right)^{0.49}$ (Amati 2006; Li 2006), we derive $E_{\text {iso }} \gtrsim 5 \times 10^{50} \mathrm{erg}$ may be expected. Incidentally we note that our fit of the second radio peak within the off-axis GRB model implied $E_{\text {iso }} \sim 7 \times 10^{52} \mathrm{erg}$, so the two results are not in contrast with each other if one assumes a kineticenergy-to- $\gamma$-ray-energy conversion efficiency $\gtrsim 1 \%$.

We finally note that within the SSA scenario, the expected angular diameter of PTF11qcj would reach the $\sim 1$ mas level at $\sim 2500$ days post-explosion, or around $6.8 \mathrm{yr}$ since explosion. A larger angular diameter may be realized if higher ejecta speeds (such as those associated with a GRB jet) would have occurred at any time during the evolution of this explosion. Thus, late-time VLBI observations could potentially probe directly the size of PTF11qcj, and may help distinguish between SSA emission from a CSM-interacting SN, and an offaxis GRB. We also note that future VLBI observations could be useful to reveal whether a scenario completely different from the ones here proposed may account for the second radio peak in PTF11qcj light curve. For example, late-time VLBI observations of SN 1986J revealed an emerging central component whose flux density became comparable to that of the extended supernova shell about $30 \mathrm{yr}$ after explosion (Bietenholz \& Bartel 2017). Although the relevant timescales would be rather different for PTF11qcj, a scenario where the second peak may be related to a young pulsar-wind nebula or emission from an accreting black hole may be worth testing in the future.

A.C. and N.T.P. acknowledge support from the NSF CAREER award \#1455090. A.C. also acknowledges partial support by Chandra Award No. GO7-18065X. J.V. is supported by the GINOP-2.3.2-15-2016-00033 project, which is funded by the Hungarian National Research, Development and Innovation Fund and the European Union. J.C.W. is supported in part by the Samuel T. and Fern Yanagisawa Regents Professorship. A.G.-Y. is supported by the EU via ERC grant No. 725161, the Quantum Universe I-Core program, the ISF, the BSF Transformative program and by a 
Kimmel award. The National Radio Astronomy Observatory is a facility of the National Science Foundation operated under cooperative agreement by Associated Universities, Inc.

\section{ORCID iDs}

Nipuni T. Palliyaguru (i) https://orcid.org/0000-00024828-0262

Alessandra Corsi (1) https://orcid.org/0000-0001-8104-3536

\section{References}

Alexander, K. D., Laskar, T., Berger, E., et al. 2017, ApJ, 848, 69 Amati, L. 2006, MNRAS, 372, 233

Amati, L., Frontera, F., Tavani, M., et al. 2002, A\&A, 390, 81

Bartel, N., Bietenholz, M. F., Rupen, M. P., \& Dwarkadas, V. V. 2007, ApJ, 668, 924

Berger, E., Kulkarni, S. R., Frail, D. A., \& Soderberg, A. M. 2003, ApJ, 599, 408

Bietenholz, M. F., \& Bartel, N. 2007, ApJL, 665, L47

Bietenholz, M. F., \& Bartel, N. 2017, ApJ, 839, 10

Bietenholz, M. F., Bartel, N., \& Rupen, M. P. 2003, ApJ, 597, 374

Bietenholz, M. F., De Colle, F., Granot, J., Bartel, N., \& Soderberg, A. M. 2014, MNRAS, 440, 821

Blandford, R. D., \& McKee, C. F. 1976, PhFl, 19, 1130

Bromberg, O., Nakar, E., \& Piran, T. 2011, ApJL, 739, L55

Bufano, F., Pian, E., Sollerman, J., et al. 2012, ApJ, 753, 67

Cano, Z., de Ugarte Postigo, A., Pozanenko, A., et al. 2014, A\&A, 568, A19

Chandra, P., \& Frail, D. A. 2012, ApJ, 746, 156

Chevalier, R. A. 1982, ApJ, 258, 790

Chevalier, R. A. 1998, ApJ, 499, 810

Chevalier, R. A., Li, Z.-Y., \& Fransson, C. 2004, ApJ, 606, 369

Chonis, T. S., Hill, G. J., Lee, H., Tuttle, S. E., \& Vattiat, B. L. 2014, Proc. SPIE, 9147, 91470A

Chonis, T. S., Hill, G. J., Lee, H., et al. 2016, Proc. SPIE, 9908, 99084C

Corsi, A., Gal-Yam, A., Kulkarni, S. R., et al. 2016, ApJ, 830, 42

Corsi, A., Ofek, E. O., Gal-Yam, A., et al. 2014, ApJ, 782, 42

D’Elia, V., Pian, E., Melandri, A., et al. 2015, A\&A, 577, A116

Eichler, D., \& Levinson, A. 1999, ApJL, 521, L117

Ensman, L. M., \& Woosley, S. E. 1988, ApJ, 333, 754

Frail, D. A., Waxman, E., \& Kulkarni, S. R. 2000, ApJ, 537, 191

Fransson, C., \& Björnsson, C.-I. 1998, ApJ, 509, 861

Fynbo, J. P. U., Watson, D., Thöne, C. C., et al. 2006, Natur, 444, 1047

Galama, T. J., Vreeswijk, P. M., van Paradijs, J., et al. 1998, Natur, 395, 670

Gal-Yam, A. 2017, in Handbook of Supernovae, Observational and Physical Classification of Supernovae (Cham: Springer International), 195

Gal-Yam, A., Fox, D. B., Price, P. A., et al. 2006, Natur, 444, 1053

Garmire, G. P., Bautz, M. W., Ford, P. G., Nousek, J. A., \& Ricker, G. R., Jr. 2003, Proc. SPIE, 4851, 28

Granot, J., \& Ramirez-Ruiz, E. 2004, ApJL, 609, L9

Högbom, J. A. 1974, A\&AS, 15, 417

Kulkarni, S. R., Frail, D. A., Wieringa, M. H., et al. 1998, Natur, 395, 663

Kumar, P., \& Panaitescu, A. 2000, ApJL, 541, L51
Laskar, T., Berger, E., Margutti, R., et al. 2015, ApJ, 814, 1

Laskar, T., Berger, E., Tanvir, N., et al. 2014, ApJ, 781, 1

Law, N. M., Kulkarni, S. R., Dekany, R. G., et al. 2009, PASP, 121, 1395 Li, L.-X. 2006, MNRAS, 372, 1357

Li, Z.-Y., \& Chevalier, R. A. 1999, ApJ, 526, 716

Malesani, D., Tagliaferri, G., Chincarini, G., et al. 2004, ApJL, 609, L5

Matheson, T., Garnavich, P. M., Stanek, K. Z., et al. 2003, ApJ, 599, 394

Mazzali, P. A., Deng, J., Pian, E., et al. 2006, ApJ, 645, 1323

Mazzali, P. A., Deng, J., Tominaga, N., et al. 2003, ApJL, 599, L95

Mazzali, P. A., McFadyen, A. I., Woosley, S. E., Pian, E., \& Tanaka, M. 2014, MNRAS, 443, 67

McMullin, J. P., Waters, B., Schiebel, D., Young, W., \& Golap, K. 2007, in ASP Conf. Ser. 376, Astronomical Data Analysis Software and Systems XVI, ed. R. A. Shaw, F. Hill, \& D. J. Bell (San Francisco, CA: ASP), 127

Milisavljevic, D., Margutti, R., Kamble, A., et al. 2015, ApJ, 815, 120

Modjaz, M., Liu, Y. Q., Bianco, F. B., \& Graur, O. 2016, ApJ, 832, 108

Paczyński, B. 1998, ApJL, 494, L45

Paczynski, B. 2001, AcA, 51, 1

Paragi, Z., Taylor, G. B., Kouveliotou, C., et al. 2010, Natur, 463, 516

Perna, R., \& Loeb, A. 1998, ApJL, 509, L85

Pian, E., Mazzali, P. A., Masetti, N., et al. 2006, Natur, 442, 1011

Pignata, G., Stritzinger, M., Soderberg, A., et al. 2011, ApJ, 728, 14

Piran, T. 2004, RvMP, 76, 1143

Quimby, R., Rykoff, E., \& Yuan, F. 2007, CBET, 927, 1

Rau, A., Kulkarni, S. R., Law, N. M., et al. 2009, PASP, 121, 1334

Rhoads, J. E. 1997, ApJL, 487, L1

Roy, N., Bharadwaj, S., Dutta, P., \& Chengalur, J. N. 2009, MNRAS, 393, L26

Salas, P., Bauer, F. E., Stockdale, C., \& Prieto, J. L. 2013, MNRAS, 428, 1207

Schulze, S., Malesani, D., Cucchiara, A., et al. 2014, A\&A, 566, A102

Smith, N. 2014, ARA\&A, 52, 487

Soderberg, A. M., Brunthaler, A., Nakar, E., Chevalier, R. A., \& Bietenholz, M. F. 2010, ApJ, 725, 922

Soderberg, A. M., Chakraborti, S., Pignata, G., et al. 2010, Natur, 463, 513

Soderberg, A. M., Chevalier, R. A., Kulkarni, S. R., \& Frail, D. A. 2006, ApJ, 651,1005

Soderberg, A. M., Kulkarni, S. R., Berger, E., et al. 2005, ApJ, 621, 908

Soderberg, A. M., Nakar, E., Berger, E., \& Kulkarni, S. R. 2006, ApJ, 638, 930

Sparre, M., Sollerman, J., Fynbo, J. P. U., et al. 2011, ApJL, 735, L24

Stanek, K. Z., Matheson, T., Garnavich, P. M., et al. 2003, ApJL, 591, L17

Taddia, F., Sollerman, J., Leloudas, G., et al. 2015, A\&A, 574, A60

Taylor, G. I. 1946, RSPSA, 186, 273

van Dyk, S. D., Weiler, K. W., Sramek, R. A., \& Panagia, N. 1993, ApJL, 419, L69

van Eerten, H., van der Horst, A., \& MacFadyen, A. 2012, ApJ, 749, 44

van Eerten, H., Zhang, W., \& MacFadyen, A. 2010, ApJ, 722, 235

Waxman, E. 2004, ApJ, 602, 886

Weiler, K. W., Panagia, N., \& Sramek, R. A. 1990, ApJ, 364, 611

Weiler, K. W., Sramek, R. A., Panagia, N., van der Hulst, J. M., \& Salvati, M. 1986, ApJ, 301, 790

Wellons, S., Soderberg, A. M., \& Chevalier, R. A. 2012, ApJ, 752, 17

Wood-Vasey, W. M., Aldering, G., Nugent, P., \& Chassagne, R. 2003, IAUC, 8082,1

Woosley, S. E., \& Bloom, J. S. 2006, ARA\&A, 44, 507

Xu, D., de Ugarte Postigo, A., Leloudas, G., et al. 2013, ApJ, 776, 98

Yamazaki, R., Yonetoku, D., \& Nakamura, T. 2003, ApJL, 594, L79

Zhang, W., \& MacFadyen, A. 2009, ApJ, 698, 1261 